\title{
Super-elastic Collision of Large-scale Magnetized Plasmoids in The Heliosphere
}

\author{
Chenglong Shen ${ }^{1}$, Yuming Wang ${ }^{1, *}$, Shui Wang ${ }^{1}$, Ying Liu ${ }^{2,4}$, Rui Liu ${ }^{1}$, Angelos Vourlidas ${ }^{3}$, \\ Bin Miao ${ }^{1}$, Pinzhong $\mathrm{Ye}^{1}$, Jiajia Liu ${ }^{1}$, and Zhenjun Zhou ${ }^{1}$ \\ ${ }^{1}$ CAS Key Laboratory of Geospace Environment, Department of Geophysics and Planetary Sciences, University of Science EG \\ Technology of China, Hefei 230026, China \\ ${ }^{2}$ Space Sciences Laboratory, University of California, Berkeley, CA 94720, USA \\ ${ }^{3}$ Space Science Division, Naval Research Laboratory, Washington, D.C. 20375, USA \\ ${ }^{4}$ State Key Laboratory of Space Weather, National Space Science Center, Chinese Academy of Sciences, Beijing, China \\ *Corresponding author. E-mail: ymwang@ustc.edu.cn
}

\begin{abstract}
Super-elastic collision is an abnormal collisional process, in which some particular mechanisms cause the kinetic energy of the system increasing. Most studies in this aspect focus on solid-like objects, but they rarely consider gases or liquids, as the collision of the latter is primarily a mixing process. With cross-field diffusion being effectively prohibited, magnetized plasmoids are different from ordinary gases. But it remains unclear how they act during a collision. Here we present the global picture of a unique collision between two coronal mass ejections in the heliosphere, which are the largest magnetized plasmoids erupting from the Sun. Our analysis for the first time reveals that these two magnetized plasmoids collided like solid-like objects with a $73 \%$ likelihood of being super-elastic. Their total kinetic energy surprisingly increased by about $6.6 \%$ through the collision, which significantly influenced the dynamics of the plasmoids.
\end{abstract}

\section{Introduction}

Collisional dynamics is essential in determining global structure and evolution of macro- and micro- objects, like planet rings 1, granular materials 2, and nanoclusters 3, 4]. To classify collisions in terms of energy transfer, Newton defined the coefficient of restitution, $e$, which is normally between 0 and 1 . However, abnormal $e$ values, such as $e>1$ (ref. [2, 5, 6, 7]) or $e<0$ (ref. 44) have been reported. A super-elastic collision is a process through which the linear kinetic energy of the collisional system increases, i.e., $|e|>1$. In the literature, there have been several mechanisms proposed to explain such an abnormal increase of linear kinetic energy during a collision. In granular physics, for example, the oblique impact collision with local deformation may help transfer rotational kinetic energy into linear kinetic energy 2, 5, 4] (hereafter kinetic energy refers to linear kinetic energy). Thermal fluctuations are suggested as another possible reason leading to super-elastic collisions of nanoclusters 3 .

In absence of internal magnetic fields, two encountering plasmoids tend to mix together, just like ordinary gases. But it is unclear what would happen if they carry strong magnetic fields, especially in regards to the nature of collision and the energy exchange between them. Coronal mass ejections (CMEs) are large-scale 8 magnetized plasmoids, originating from the solar atmosphere and expanding and propagating into the heliosphere. Since they are a frequently-occurring phenomenon with an occurrence rate of $4-5$ CMEs per day during solar maximum 9 , the encounters and interactions between CMEs are unavoidable. Actually, as a consequence of interactions, multipleinterplanetary-CME structures are often observed by in situ instruments 10, 11, 12, 13, 14. Thus the issue of magnetized plasmoid collision may be addressed by investigating observations of CMEs.

However, the CME dynamics in the heliosphere constitute an intricate problem 15, 16, 17, 18, especially when the collision/interaction between CMEs is involved 19, 11, 20, 21. 22. The dynamics of two successive CMEs of $24-25$ January 2007 was discussed by Lugaz et al. 21. They proposed four different scenarios to explain observations, one of which they think is a mysterious collision through which the leading CME gained momentum and finally became faster than the overtaking CME. Most recently, a CME-CME interaction event of 1 August 2010 has been intensively studied with a focus on the CME dynamics, CME-driven shock and radio bursts 22, 23, 24. Numerical simulations of the interaction between CMEs were also carried out by many researchers 25, 26, 27, 28, 29, 30, 31, 32, but few discussed the nature of the CME collisions.

During $2-8$ November 2008, the Sun Earth Connection Coronal and Heliospheric Investigation (SECCHI) suites 33 onboard the twin Solar TErrestrial RElations Observatories (STEREO) 34 captured the process of the chasing and colliding of two CMEs in the heliosphere with clear imaging observations. Each SECCHI suite carries the cameras COR1, COR2, HI1 and HI2, and can seamlessly track CMEs from the corona to interplanetary space. Since the events occurred near the solar minimum, the conditions in the heliosphere were quite simple. The events provide us with a unique opportunity to study the physical details of CME col- 
lisions. As will be seen, the collision between the two CMEs is super-elastic in nature, during which their total kinetic energy increased. These results advance our understanding of the behavior of large-scale magnetized plasmoids.

\section{Imaging of two successive CMEs and their collision}

The two CMEs originated from the Sun at about 00:35 UT and 22:35 UT, respectively, on 2 November 2008, when STEREO-A spacecraft was located at $0.97 \mathrm{AU}$ and $41^{\circ}$ to the west of the Sun-Earth line, while STEREO-B was located at $1.07 \mathrm{AU}$ and $40^{\circ}$ to the east (Fig 14). These events were reported by Kilpua et al. 35 with a focus on their solar source locations and in situ effects at $1 \mathrm{AU}$. One can refer to that paper or Sec.2 of Supplementary Information for the details of the propagation of the two CMEs in the corona. Here we focus on their collision in the heliosphere.

Being faster than CME1, CME2 finally caught up and collided with CME1. This phenomenon was clearly recorded by HI1 onboard STEREO-B, or HI1-B briefly. Based on the HI1-B images, we can see that the distance between the front edge of CME2 and the rear edge of CME1 became smaller and smaller. The apparent touch of the two CMEs began approximately around 18:49 UT on 3 November 2008, which was registered as a significant enhancement of brightness around an arc-shaped structure (Fig $2 \mathrm{~d})$. We call the brightness enhanced region as collision region, and the arc structure is the core of the region. Since the arc structure is caving into CME2, the brightness enhancement is not simply due to superposition of the two CMEs, but probably the result of a soft object colliding with a hard object. Actually, if the two CMEs did not collide, the kinetic evolution of CME1 cannot be explained only by solar wind acceleration (refer to Sec.11 of Supplementary Information). The brightened arc structure stayed visible for about 7 hours with the most clear appearance at around 00:09 UT on 4 November (Fig 2r). The whole collision region remained brightened much longer till 10:49 UT on 4 November 2008 (Fig22). It seems that the entire collisional process of such large-scale magnetized plasmoids is similar to that of elastic balls, which includes a pre-collision phase, a compression phase, a restitution phase and a post-collision phase. We think that the appearance and disappearance of the visible arc structure define the start and the end of the compression phase, respectively, and the complete disappearance of the brightened region between the two CMEs marks the end of the restitution phase, i.e., the end of the collision between them. The movies are available as online materials.

\section{Tracking and Dynamics of the two CMEs in the heliosphere}

In order to analyze the dynamics of the CMEs and their collision, a time-elongation map, known as Jmap 36, 37, 17 38 , is constructed. To facilitate the comparison between imaging data and in situ data at $1 \mathrm{AU}$, a 64-pixel wide slice is placed along the ecliptic plane in the running-difference images from COR2, HI1 and HI2 onboard STEREO-B to produce the Jmap (Fig 31). A bright-dark alternating track from lower-left to upper-right usually indicates a bright structure moving away from the Sun. The two vertical dotted green lines mark the start and end times of the collision.

The front edges of CME1 and CME2 are distinct in the Jmap as marked by the red and blue ' $\diamond$ ', respectively. They are the same points marked by the red and blue ' $\diamond$ ' in Fig 2 a and 20. The rear edges of the two CMEs are not clear in the Jmap. To find out where the tracks of the rear edges of the two CMEs are, we directly identify their rear edges in coronagraph images like in Fig 2a and 20, and then dot them back to the Jmap as shown by the red and blue ' + ', respectively. Note that the significant track between the red ' $\diamond$ ' and ' + ' symbols does not correspond to the CME1's rear edge but to its bright core.

The elongation angle of a given feature in the Jmap can be converted to the heliocentric distance under some assumptions 39, 40, 17, 38. An often used assumption is to approximate a CME as a sphere 21, 32]. By further assuming that the front and rear edges recorded in the Jmap are the points of tangency determined by the circular crosssection of the CME in the ecliptic plane and the observer STEREO-B, we get the heliocentric distance of the CME center, $d$, its radius $r$, and their projected components on the ecliptic plane, $d_{p}$ and $r_{p}$, in terms of the heliocentric distance, $l$, of STEREO-B, the elongation angles, $\varepsilon_{F}$ and $\varepsilon_{R}$, of the CME front and rear edges, and the latitude, $\theta$, and longitude, $\varphi$, of the CME center. The detailed derivation can be found in the Sec.4 of Supplementary Information. Due to the presence of the solar wind stretching effect, a CME might become 'pancake' shaped even if it was initially spherical 41, 42. The HI1 imaging data suggests that the effect is somewhat significant for CME2, but not for CME1. Thus a small correction is made to CME2 to reduce the effect (refer to Sec.5 of Supplementary Information).

With the aid of the Graduated Cylindrical Shell (GCS) model 43, 44, the latitude, $\theta$, and longitude, $\varphi$, of the two CME centers can be obtained from COR2 images. It is found that both CMEs propagated almost radially with a nearly constant longitude and latitude in the COR2 FOV 35 , which are listed in Table 1(refer to Sec.2 and 3 of Supplementary Information for details). As the interplanetary magnetic field and solar wind density get weaker and lower, respectively, farther away from the Sun, it is reasonable to assume that they would keep their propagation directions in the HI1 FOV until the collision. The results given by the model suggest that both CMEs propagated between the Sun-Earth line and the Sun-STEREO-A line with CME1 closer to the latter line and CME2 closer to the former, which is in agreement with the previous study 35 .

Figure 4 shows $d$ and $r$ as a function of time for both CMEs. Since the front and rear edges of the CMEs are more or less diffused, a reasonable error of $\pm 5 \%$ in determination of the elongation angle of the CME front and rear edges is considered. The resultant undertainties of $d$ and $r$ are indicated by the error bars in Figure 4 By applying the linear fitting to $d$ and $r$ with these uncertainties taken into account, we get the propagation speed $v_{c}$ and expansion speed $v_{e}$ of the two CMEs, as well as their components in the ecliptic plane, $v_{p}$ and $v_{e p}$. A 2- $\sigma$ uncertainty of the speeds derived from the linear fitting is applied in the following analysis. The excellent consistency between the fitting lines and the data points suggests that the two CMEs experienced a nearly constant-speed propagation and expansion 

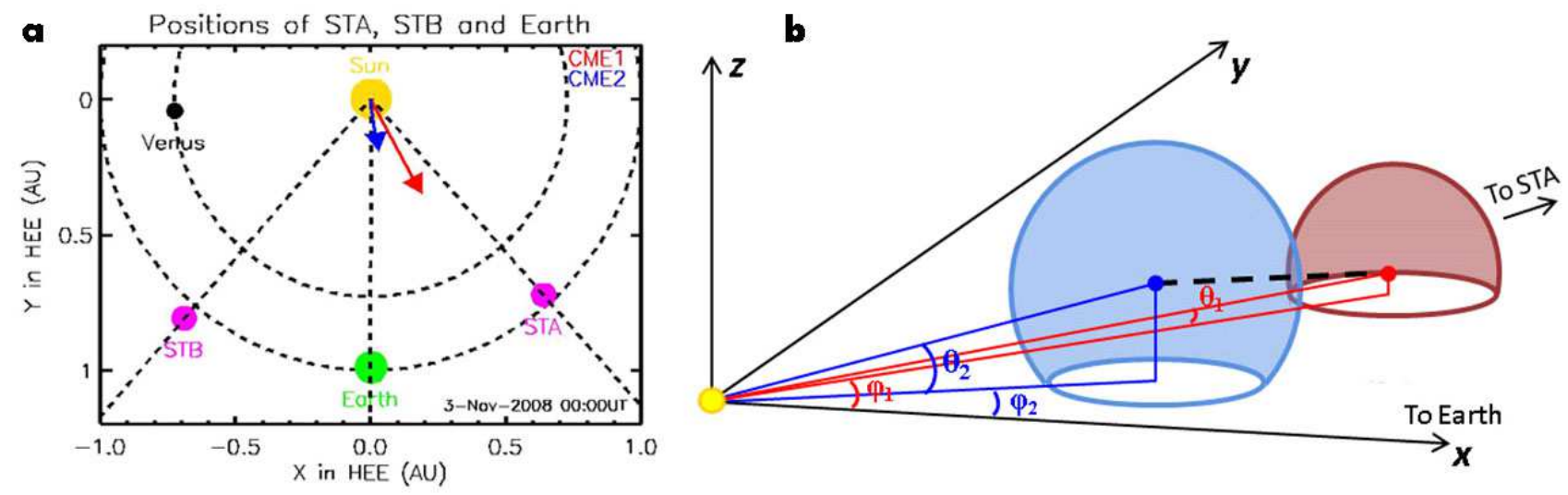

Figure 1: The sketch map of (a) the positions of spacecraft and (b) the collision of the CMEs.
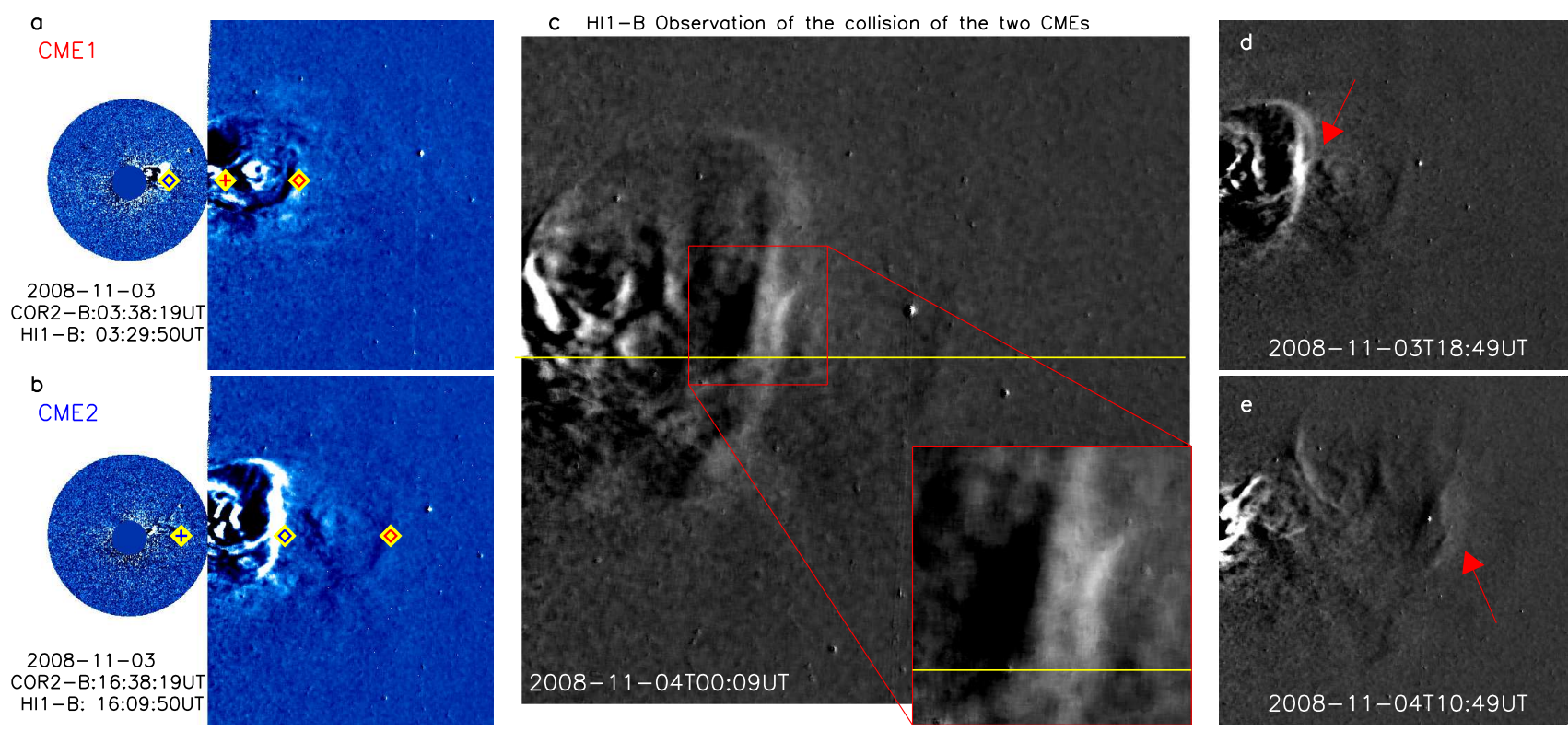

Figure 2: The STEREO/SECCHI images of the two CMEs and their collision in the heliosphere. (a) and (b) are the running-difference images showing CME1 and CME2. The red symbol ' $\diamond$ ' and ' + ' mark the front and rear edges of CME1, respectively, and the blue symbols for CME2. (c) The running-difference image of HI1-B showing the collision of the two CMEs. (d) and (e) show the beginning and end of the collision, and the red arrows indicate the collision region. 


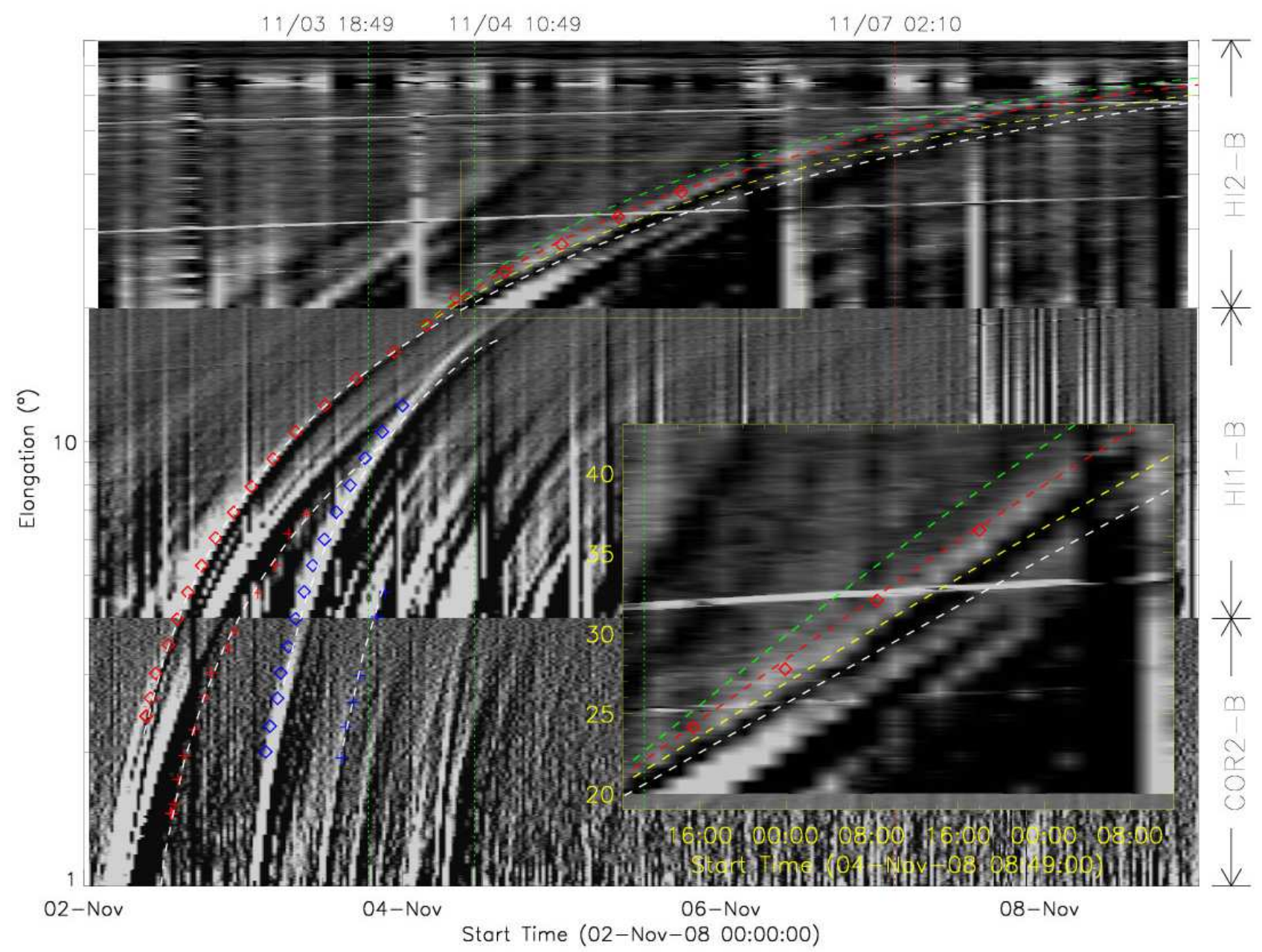

Figure 3: The time-elongation map from 2 to 9 November 2008 constructed based on the running-difference images from STEREO-B. The symbol of ' $\diamond$ ' and ' + ' show the front and rear edges of the CMEs, respectively. The two vertical dotted green lines indicate the start and end of the collision. The red vertical line marks the arrival time of CME1 at STEREO-A. The region enclosed by yellow rectangle is zoomed-in in the lower-right corner. Refer to the main text for more details.

Table 1: The parameters of the two CMEs before and after the collision.

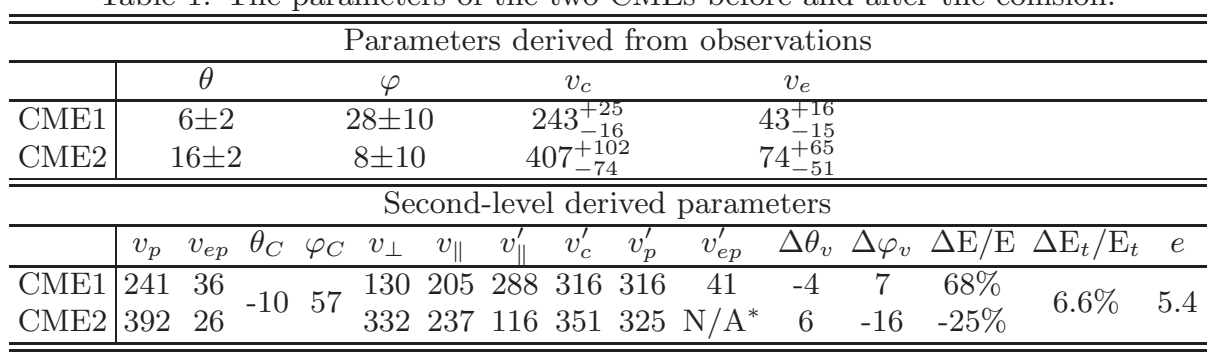

$\theta$ and $\varphi$ are the CME's latitude and longitude. $v_{c}$ and $v_{e}$ are the propagation and expansion speed of a CME, derived from the Jmap by assuming the CME is a sphere (refer to Sec.4 and 5 of Supplementary Information). $v_{p}$ and $v_{e p}$ are the average values of the components of $v_{c}$ and $v_{e}$ in the ecliptic plane, respectively. $\theta_{C}$ and $\varphi_{C}$ are the latitude and longitude of the collision direction (refer to Fig.7 in Sec.7 of

Supplementary Information). $v_{\perp}$ and $v_{\|}$are the components of the CME velocity perpendicular and parallel to the collision direction, respectively. The superscript of prime denotes the parameters after the collision. $\Delta \theta_{v}$ and $\Delta \varphi_{v}$ are the change of the CME velocity.

$\Delta E / E=\left(E^{\prime}-E\right) / E$ is the percentage of the kinetic energy changed, and $E_{t}$ is the sum of the kinetic energy of the two CMEs. All the angles in the table are in units of degree, and all the speeds are in units of $\mathrm{km} \mathrm{s}^{-1}$. Here, only the uncertainties of $\theta, \varphi, v_{c}$ and $v_{e}$ are listed, and the uncertainties of speeds have included the uncertainties in the CMEs' directions. The uncertainties of the second-level derived parameters are not listed, but all taken into account in our analysis. * After the collision, CME2 left the ecliptic plane, and thus there is no available component of expansion speed in the ecliptic plane. 
in the heliosphere before they encountered, though a very weak acceleration can be seen for CME1. It should be noted that the uncertainties of CMEs' directions may cause additional uncertainties of CMEs' speeds, and therefore the final values of the uncertainties of CMEs' speeds (see $v_{c}$ and $v_{e}$ listed in Table 1) are larger than those given in Figure 4 Besides, although the front edge of CME2 perhaps traveled faster than background solar wind, observations suggest that it did not drive an evident shock ahead (refer to Sec.12 of Supplementary Information).

Further, we reversely derive the elongation angle-time curves from the above results, and plot them on the Jmap in white dashed lines in Figure 3 These white dashed lines are also extrapolated to the post-collision phase. It is found that the fitting lines match the observed tracks very well before the collision, but begin to deviate from the tracks since the beginning of the collision (particularly note the tracks of the two CMEs' front edges). Such deviations mean that the collision between the two CMEs must have significantly changed their propagation directions and/or speeds.

As an attempt, we might as well treat the CMEs approximately as a expanding ball in the collision. The situations of the two CMEs at the time of touching have been sketched in Figure 1b. It is a collision in 3-D space. Since CME1 was originally propagating along a radial direction with lower latitude than CME2, the collision should push CME1 closer to the Sun-STEREO-A line in the ecliptic plane and CME2 further away from the ecliptic plane. Thus, it is expected that CME1 would be observed in situ by the instruments onboard STEREO-A while CME2 would be missed by the in situ instruments which are all located in the ecliptic plane. The in situ data at 1 AU do suggest that only CME1 was observed as expected (refer to Sec.6 of Supplementary Information for more details). Its propagation and expansion speeds at $1 \mathrm{AU}$ were about 342 and $30 \mathrm{~km} \mathrm{~s}^{-1}$, respectively. The increased propagation speed is consistent with our conjecture that CME1 was accelerated by the collision. The expansion speed is very close to that derived from Jmap. This fact allows us to reasonably assume that the expansion speed was recovered after the collision for both CMEs, though the expansion speed may vary greatly during the collision and CME2 was not locally observed at $1 \mathrm{AU}$.

\section{Super-elastic collision and the energy ex- change}

Imagining two expanding elastic balls, not only their collision will result in the momentum exchange in the direction connecting the centroids of the two balls (referred to as collision direction hereafter), but also their continuous expansion may cause their centroids to separate farther away. We define the approaching speed as the speed of the centroid of one ball relative to the other in the collision direction. Under the assumption that the expansion speeds remained unchanged before and after collision, the collision should be super-elastic if the sum of the expansion speeds of the two balls was larger than the approaching speed before the collision. Here we first show the results for the case of the CMEs' parameters given in Table 1 and then analyze the influence of the uncertainties.

According to the values listed in Table 1 we can derive that the latitude $\theta_{C}$ and longitude $\varphi_{C}$ of the collision direction at the beginning of the collision, i.e., the elevation angle and azimuthal angle in the heliocentric coordinate system, are about $-10^{\circ}$ and $57^{\circ}$, respectively. By resolving the propagation velocity vectors into the components parallel, $v_{\|}$, and perpendicular, $v_{\perp}$, to the collision direction (refer to Sec.7 of Supplementary Information), we find that the values of $v_{\|}$of the two CMEs were 205 and $237 \mathrm{~km} \mathrm{~s}^{-1}$, respectively (listed in Table 1), which give an approaching speed of about $32 \mathrm{~km} \mathrm{~s}^{-1}$. The sum of the expansion speeds of the two CMEs was about $117 \mathrm{~km} \mathrm{~s}^{-1}$, much larger than the approaching speed. Hence a super-elastic collision is expected.

The conservation of momentum requires $m_{1} v_{1 \|}+m_{2} v_{2 \|}=$ $m_{1} v_{1 \|}^{\prime}+m_{2} v_{2 \|}^{\prime}$, where $m_{1}$ and $m_{2}$ are the mass of CME1 and CME2, respectively, and the prime symbol denotes the parameters after the collision. Here, we approximately treat the collision phase including the compression and restitution phases as a black box, and adopt parameters of the two CMEs before (after) the collision for the first (second) half period of the collision phase. The influence of this simplification on our final result is not significant (see Sec. 8 of Supplementary Information).

The mass of a CME can be calculated from calibrated coronagraph images 45. For CME1 and CME2, the derived masses based on COR2-B observations are about $1.8 \times 10^{12}$ $\mathrm{kg}$ and $1.2 \times 10^{12} \mathrm{~kg}$, respectively. The Thomson scattering and projection effects have been corrected 46, 47. The mass ratio of CME1 to CME2 is about 1.5. Hence, for any given coefficient of restitution $e$, i.e., $\frac{v_{2 \|}^{\prime}-v_{1 \|}^{\prime}}{v_{1 \|}-v_{2 \|}}$, the velocities of the two CMEs after the collision can be obtained (refer to Sec.7 of Supplementary Information) as well as the expected tracks of the front and rear edges of both the CMEs in the Jmap. Actually, our calculation suggests that, no matter which value of the mass ratio we choose, the super-elastic nature of the collision, which will be seen below, does not change (refer to Sec.9 of Supplementary Information).

In the Jmap, only the track of the front edge of CME1 is still identifiable after the collision. Thus we repeatedly adjust the value of $e$ to find the best match for the observed track. For the parameters listed in Table 1 (the influence of the uncertainties will be addressed in the last paragraph of this section), the red dashed lines starting at the middle of the collision in Figure 3 shows the best predicted track of the front edge of CME1, which gives $e=5.4$. As a comparison, the tracks for $e$ equal to 1 and 10 are presented by the yellow and green dashed lines, respectively. A zoomedin image in the lower-left corner of Figure 3 presents the details. Obviously, the tracks predicted by both the yellow and green dashed lines get worse. $e=1$ indicates a perfect elastic collision, but the yellow line is obviously lower than the observed track indicated by the red ' $\diamond$ '. The $0<e<1$ tracks predicted by our calculation would be located even lower.

As summarized in Table 1 through the collision, CME1 was deflected southwestward and its propagation speed increased from $243 \mathrm{~km} \mathrm{~s}^{-1}$ to about $316 \mathrm{~km} \mathrm{~s}^{-1}$, while CME2 was deflected northeastward and its speed decreased from $407 \mathrm{~km} \mathrm{~s}^{-1}$ to $351 \mathrm{~km} \mathrm{~s}^{-1}$. The in situ propagation speed of CME1 was about $40 \mathrm{~km} \mathrm{~s}^{-1}$ larger than the derived postcollision speed of CME1. It is probably due to the continuous 


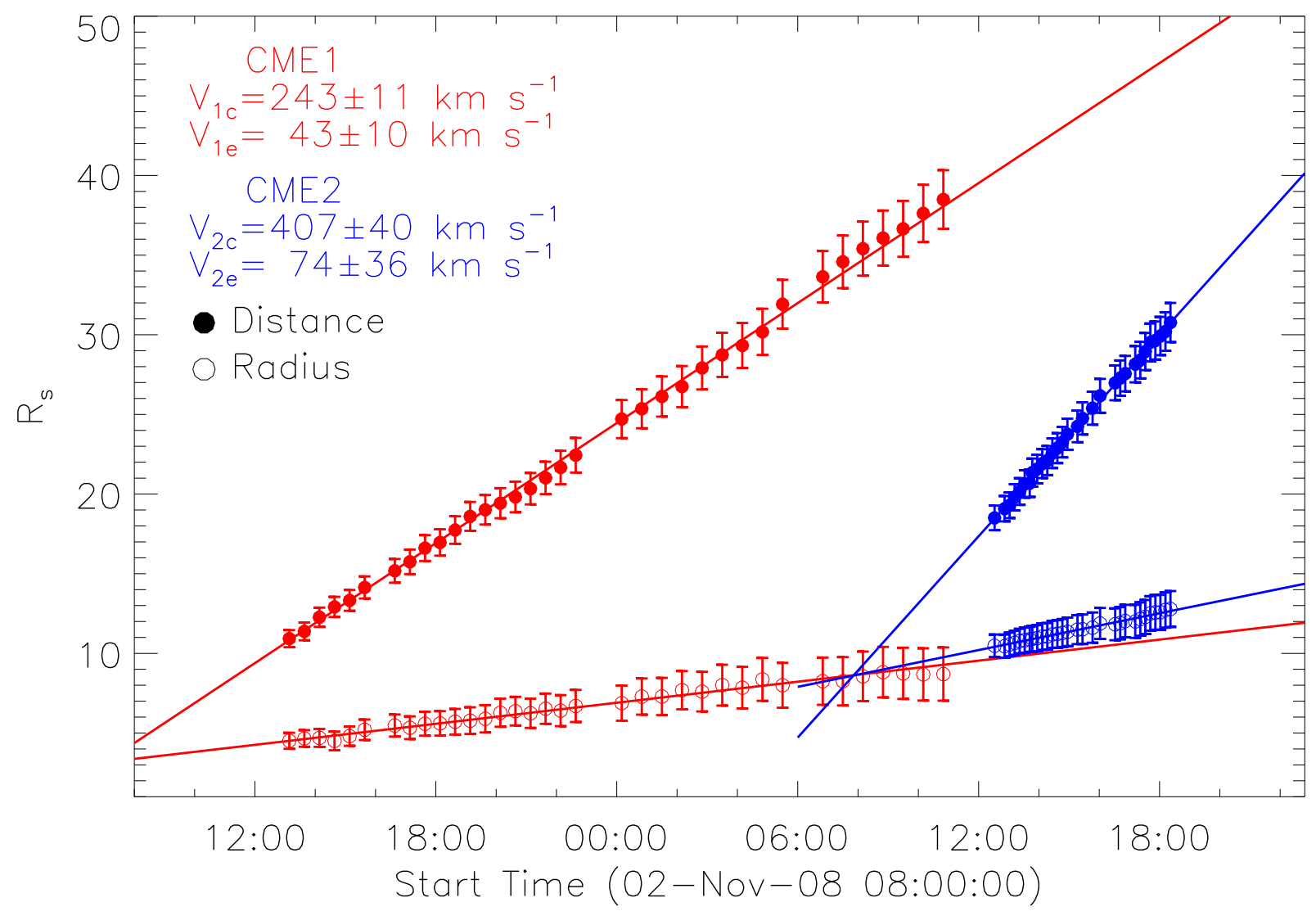

Figure 4: The heliocentric distance $d$ (dots) and the radius $r$ (open circles) of the two CMEs as functions of time for the case that $\theta$ and $\varphi$ of CME1 are $6^{\circ}$ and $28^{\circ}$, respectively and those of CME2 are $16^{\circ}$ and $8^{\circ}$, respectively. The error bars are derived from the $5 \%$ uncertainty in elongation angle. Speeds are obtained by linear fitting with the error bars taken taken into account, and a $2-\sigma$ uncertainty is chosen, which makes the confidence level greater than $95 \%$. 
acceleration by the solar wind. According to the result, the two CMEs were separating after the collision (refer to Sec.10 of Supplementary Information for a preliminary discussion). It is worth noting that CME2 is completely above the ecliptic plane after the collision. Therefore, it is not surprising that no counterpart of CME2 was detected by in situ instruments located in the ecliptic plane. Further, the kinetic energy of CME1 (the contribution from the CME expansion has been taken into account) is found to increase by about $68 \%$, while that of CME2 decreased by about $25 \%$. As a whole, the system gained about $6.6 \%$ kinetic energy during the collision.

The influence of large uncertainties, i.e., those in the CMEs' longitudes and velocities as listed in Table 1 is further examined. We sample the longitudes of the two CMEs at $1^{\circ}$ within the $10^{\circ}$ uncertainty. For each possible pair of longitudes we consider an combination of five propagation speeds, $\left[v_{c} \pm \Delta_{v c}, v_{c} \pm 0.5 \Delta_{v c}, v_{c}\right]$, for either of both CMEs and five expansion speeds, $\left[v_{e} \pm \Delta_{v e}, v_{e} \pm 0.5 \Delta_{v e}, v_{e}\right]$, for CME1, which constitute 125 cases. Here, $\Delta_{v c}$ and $\Delta_{v e}$ are the uncertainties in the CME speeds. For each case we are able to obtain a value of $e$ and the change of the total kinetic energy. The likelihood of super-elastic collision for each longitude pair is therefore calculated. Figure 5 presents the result. Most area shows a strong likelihood of super-elastic collision. Specifically, $72.6 \%$ are more than $75 \%$ likely, and $63.0 \%$ are $100 \%$ likely, to experience a super-elastic collision. In contrast, as a few as $6.3 \%$ combinations are definitely nonsuper-elastic. Overall, it is $72.8 \%$ likely for the collision to be super-elastic.

\section{$5 \quad$ Source of kinetic energy gain}

The source of the net kinetic energy gain and the way/condition of the energy conversion are key issues for super-elastic collisions. The divergent configuration of solar wind implies that the internal pressure of a CME is always stronger than the external pressure when it move away from the Sun, which is the main cause of the CME expansion. In this process, the magnetic and thermal energies of the CME are continuously dissipated [48, 49. It could be estimated that, for a typical CME at $1 \mathrm{AU}$ with the magnetic field strength of $10 \mathrm{nT}$, temperature of $10^{5} \mathrm{~K}$, density of 5 $\mathrm{cm}^{-3}$ and velocity of $500 \mathrm{~km} \mathrm{~s}^{-1}$, the magnetic and thermal energy density is about $6 \%$ of the kinetic energy density. The percentage will be much higher when the CME is closer to the Sun. Thus, the magnetic and thermal energy of CMEs should be sufficient to provide $\mathrm{a} \sim 6.6 \%$ increase of kinetic energy in the super-elastic collision, and the persistent expansion of CMEs may provide the way for the magnetic/thermal energy to convert into kinetic energy.

Besides, the detailed interacting process may be also important in determining the nature of collision. An anticorrelation between the impact velocity and the coefficient of restitution was reported in collisions among ice particles of Saturn B ring [1 and granular materials 50. Experiments and simulations on granular materials and nanoclusters have further shown that the collision between a hard sphere and a soft plate tends to be super-elastic 2, 5, 4, These imply that super-elastic collision requires sufficient interaction time and touching area for momentum exchange and energy conversion. In our case, the compression and restitution phases lasted about 16 hours, during which a clear arc-shaped structure stayed visible for about 7 hours. These phenomena suggest that the two CMEs had sufficient time and sufficiently large touching area to convert magnetic/thermal energy into kinetic energy. It is worthy of further investigation to see if a similar anti-correlation applies to CME collisions, i.e., larger coefficient of restitution corresponds lower impact velocity.

Although in granular physics, rotational motion and thermal fluctuation have been considered the possible mechanism for the increased kinetic energy 2, 5, 3, 4, they are probably not suitable for CME collisions. First, there is no evidence that plasma within a CME undergoes a significant rotations in interplanetary space. Second, CMEs are a large-scale structure with huge mass and thus the thermal fluctuation of microscopic particles should not be able to affect the macroscopic behavior of CMEs.

In summary, the good match between the predictions by the simplest collision model and the observations suggests that such large-scale magnetized plasmoids could be simplified as balls instead of using complicated magnetohydrodyanmics or plasma kinetic theories in studying their collision. The collision may be super-elastic, through which the system gains kinetic energy from the magnetic/thermal energy of CMEs. Of course, the process and consequence might be different if significant reconnection occurs in the collision region. This will be another issue.

\section{References}

[1] Bridges, F. G., Hatzes, A. \& Lin, D. N. C. Structure, stability and evolution of Saturn's rings. Nature 309, 333-335 (1984).

[2] Louge, M. Y. \& Adams, M. E. Anomalous behavior of normal kinematic restitution in the oblique impacts of a hard sphere on an elastoplastic plate. Phys. Rev. E 65, 021303 (2002).

[3] Kuninaka, H. \& Hayakawa, H. Simulation of cohesive headon collisions of thermally activated nanoclusters. Phys. Rev. E 79, 031309 (2009).

[4] Saitoh, K., Bodrova, A., Hayakawa, H. \& Brilliantov, N. V. Negative normal restitution coefficient found in simulation of nanocluster collisions. Phys. Rev. Lett. 105, 238001 (2010).

[5] Kuninaka, H. \& Hayakawa, H. Anomalous behavior of the coefficient of normal restitution in oblique impact. Phys. Rev. Lett. 93, 154301 (2004).

[6] Smith, C. E. \& Liu, P.-P. Coefficients of restitution. J. Appl. Meteorol. 59, 963 (1992).

[7] Calsamiglia, J., Kennedy, S. W., Chatterjee, A., Ruina, A. \& Jenkins, J. T. Anomalous frictional behavior in collisions of thin disks. J. Appl. Meteorol. 66, 146 (1999).

[8] Lepping, R. P., Jones, J. A. \& Burlaga, L. F. Magnetic field structure of interplanetary magnetic clouds at 1 AU. J. Geophys. Res. 95, 11957 (1990).

[9] Yashiro, S. et al. A catalog of white light coronal mass ejections observed by the soho spacecraft. J. Geophys. Res. 109(A7), A07105 (2004).

[10] Burlaga, L. F., Plunkett, S. P. \& St. Cyr, O. C. Successive CMEs and complex ejecta. J. Geophys. Res. 107, 1266, doi:10.1029/2001JA000255 (2002).

[11] Wang, Y. M., Wang, S. \& Ye, P. Z. Multiple magnetic clouds in interplanetary space. Sol. Phys. 211, 333-344 (2002).

[12] Wang, Y. M., Ye, P. Z. \& Wang, S. Multiple magnetic clouds: Several examples during March - April, 2001. J. Geophys. Res. 108(A10), 1370, doi:10.1029/2003JA009850 (2003). 


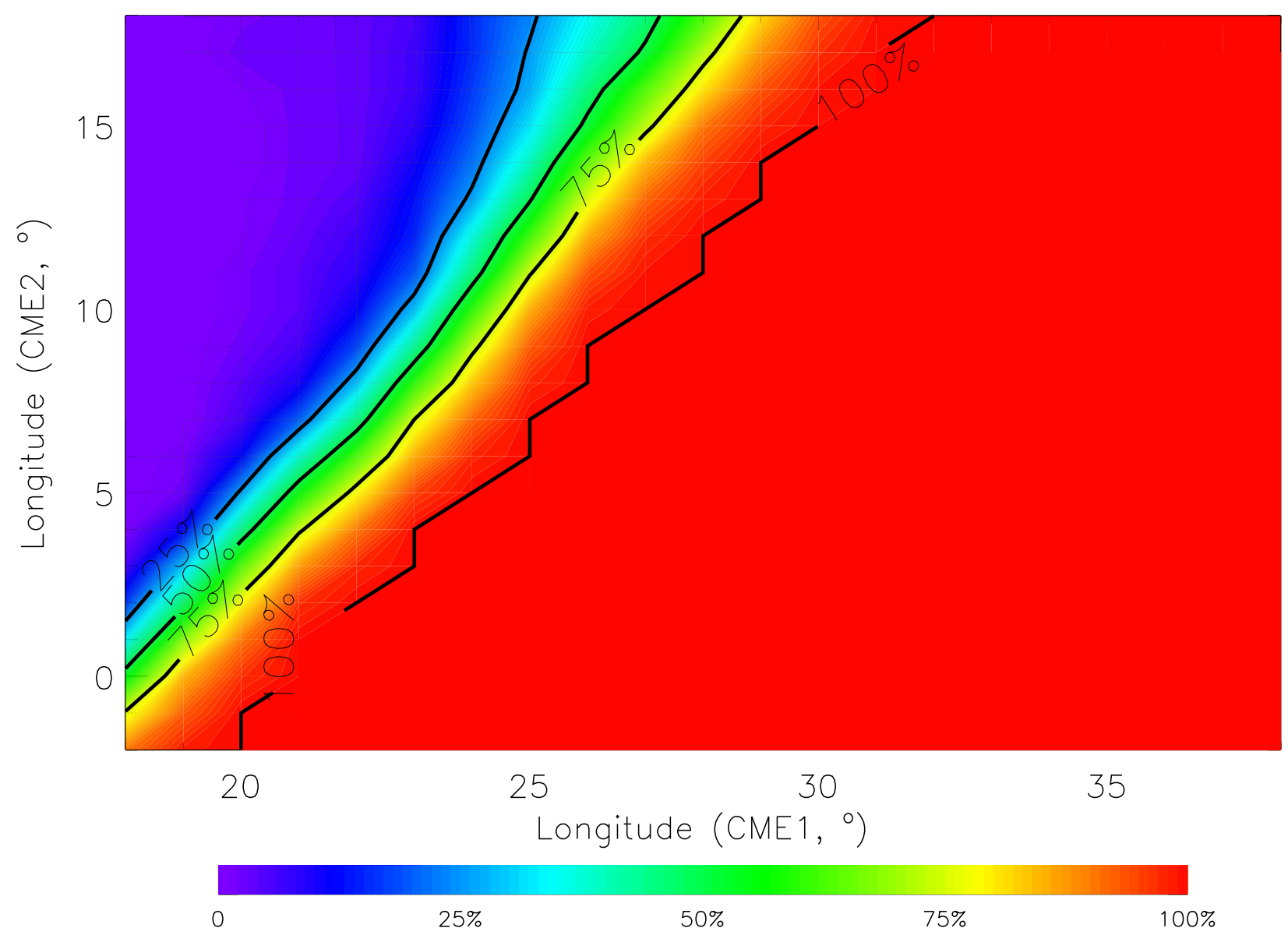

Figure 5: Possibility of super-elastic collision. See the main text for details. 
[13] Wang, Y. M., Ye, P. Z., Wang, S. \& Xue, X. H. An interplanetary cause of large geomagnetic storms: Fast forward shock overtaking preceding magnetic cloud. Geophys. Res. Lett. 30(13), 1700, doi:10.1029/2002GL016861 (2003).

[14] Farrugia, C. \& Berdichevsky, D. Evolutionary signatures in complex ejecta and their driven shocks. Annales Geophysicae 22, 3679-3698 (2004).

[15] Wang, Y. et al. Impact of the major coronal mass ejections on geo-space during September $7-13,2005$. Astrophys. J. 646, 625-633 (2006).

[16] Harrison, R. A. et al. Two years of the STEREO heliospheric imagers. invited review. Sol. Phys. 256, 219-237 (2009).

[17] Liu, Y. et al. Geometric triangulation of imaging observations to track coronal mass ejections continuously out to 1 AU. Astrophys. J. 710, L82 (2010).

[18] Liu, Y., Luhmann, J. G., Bale, S. D. \& Lin, R. P. Solar source and heliospheric consequences of the 2010 April 3 coronal mass ejection: A comprehensive view. Astrophys. J. 734, 84 (2011).

[19] Gopalswamy, N., Yashiro, S., Kaiser, M. L., Howard, R. A. \& Bougeret, J. L. Radio signatures of coronal mass ejection interaction: Coronal mass ejection cannibalism? Astrophys. J. 548, L91-L94 (2001).

[20] Shen, C., Wang, Y., Ye, P. \& Wang, S. Enhancement of solar energetic particles during a shock-magnetic cloud interacting complex structure. Sol. Phys. 252, 409-418 (2008).

[21] Lugaz, N., Vourlidas, A. \& Roussev, I. I. Deriving the radial distances of wide coronal mass ejections from elongation measurements in the heliosphere application to CME-CME interaction. Ann. Geophys. 27, 3479-3488 (2009).

[22] Liu, Y. D. et al. Interactions between coronal mass ejections viewed in coordinated imaging and in situ observations. Astrophys. J. 746, L15 (2012).

[23] Martínez Oliveros, J. C. et al. The 2010 August 01 type II burst: A CME - CME interaction, and its radio and whitelight manifestations. Astrophys. J. in press (2012).

[24] Temmer, M. et al. Characteristics of kinematics of a coronal mass ejection during the 2010 August 1 CME-CME interaction event. Astrophys. J. in press, arXiv:1202.0629 (2012).

[25] Gonzalez-Esparza, A., Santillán, A. \& Ferrer, J. A numerical study of the interaction between two ejecta in the interplanetary medium: one- and two-dimensional hydrodynamic simulations. Annales Geophysicae 22, 3741-3749 (2004).

[26] Schmidt, J. \& Cargill, P. A numerical study of two interacting coronal mass ejections. Annales Geophysicae 22, 22452254 (2004).

[27] Wang, Y., Zheng, H., Wang, S. \& Ye, P. MHD simulation of the formation and propagation of multiple magnetic clouds in the heliosphere. Astron. \&5 Astrophys. 434, 309-316 (2005).

[28] Lugaz, N., Manchester, I., W. B. \& Gombosi, T. I. Numerical simulation of the interaction of two coronal mass ejections from Sun to Earth. Astrophys. J. 634, 651-662 (2005).

[29] Hayashi, K., Zhao, X.-P. \& Liu, Y. MHD simulation of two successive interplanetary disturbances driven by cone-model parameters in IPS-based solar wind. Geophys. Res. Lett. 33, 20103 (2006).

[30] Xiong, M., Zheng, H., Wu, S. T., Wang, Y. \& Wang, S. Magnetohydrodynamic simulation of the interaction between two interplanetary magnetic clouds and its consequent geoeffectiveness. J. Geophys. Res. 112, A11103 (2007).

[31] Xiong, M., Zheng, H. \& Wang, S. Magnetohydrodynamic simulation of the interaction between two interplanetary magnetic clouds and its consequent geoeffectiveness: 2 . oblique collision. J. Geophys. Res. 114, A11101 (2009).

[32] Shen, F. et al. Three-dimensional MHD simulation of two coronal mass ejections' propagation and interaction using a successive magnetized plasma blobs model. J. Geophys. Res. 116, A09103 (2011).
[33] Howard, R. A., Moses, J., Vourlidas, A. \& et al. Sun earth connection coronal and heliospheric investigation (SECCHI). Space Sci. Rev. 136, 67-115 (2008).

[34] Kaiser, M. L. et al. The stereo mission: An introduction. Space Sci. Rev. 136, 5-16 (2008).

[35] Kilpua, E. K. J. et al. STEREO observations of interplanetary coronal mass ejections and prominence deflection during solar minimum period. Ann. Geophys. 27, 4491-4503 (2009).

[36] Sheeley, Jr., N. R., Walters, J. H., Wang, Y.-M. \& Howard, R. A. Continuous tracking of coronal outflows: Two kinds of coronal mass ejections. J. Geophys. Res. 104, 24739-24768 (1999).

[37] Davies, J. A. et al. A synoptic view of solar transient evolution in the inner heliosphere using the heliospheric imagers on STEREO. Geophys. Res. Lett. 36, L02102 (2009).

[38] Liu, Y. et al. Reconstructing coronal mass ejections with coordinated imaging and in situ observations: Global structure, kinematics, and implications for space weather forecasting. Astrophys. J. 722, 1762-1777 (2010).

[39] Lugaz, N. Accuracy and limitations of fitting and stereoscopic methods to determine the direction of coronal mass ejections from heliospheric imagers observations. Sol. Phys. 267, 411-429 (2010).

[40] Lugaz, N. et al. Determining the azimuthal properties of coronal mass ejections from multi-spacecraft remote-sensing observations with STEREO SECCHI. Astrophys. J. 715, 493-499 (2010).

[41] Riley, P. \& Crooker, N. U. Kinematic treatment of coronal mass ejection evolution in the solar wind. Astrophys. J. 600, 1035-1042 (2004).

[42] Liu, Y., Richardson, J. D., Belcher, J. W. \& Kasper, J. C. Constraints on the global structure of magnetic clouds: Transverse size and curvature. J. Geophys. Res. 111, A12S03 (2006).

[43] Thernisien, A., Howard, R. \& Vourlidas, A. Modeling of flux rope coronal mass ejections. Astrophys. J. 652, 763-773 (2006).

[44] Thernisien, A. Implementation of the graduated cylindrical shell model for the three-dimensional reconstruction of coronal mass ejections. Astrophys. J. Suppl. Ser. 194, 33 (2011).

[45] Vourlidas, A., Subramanian, P., Dere, K. P. \& Howard, R. A. Large-angle spectrometric coronagraph measurements of the energetics of coronal mass ejections. Astrophys. J. 534, 456467 (2000).

[46] Hundhausen, A. J. Sizes and locations of coronal mass ejections - SMM observations from 1980 and 1984-1989. J. Geophys. Res. 98(A8), 13177 (1993).

[47] Vourlidas, A. \& Howard, R. A. The proper treatment of coronal mass ejection brightness: A new methodology and implications for observations. Astrophys. J. 642, 1216-1221 (2006).

[48] Kumar, A. \& Rust, D. M. Interplanetary magnetic clouds, helicity conservation, and current-core flux-ropes. J. Geophys. Res. 101, 15667 (1996).

[49] Wang, Y., Zhang, J. \& Shen, C. An analytical model probing the internal state of coronal mass ejections based on observations of their expansions and propagations. J. Geophys. Res. 114, A10104 (2009).

[50] Hayakawa, H. \& Kuninaka, H. Theory of the inelastic impact of elastic materials. Phase Transitions 77, 889-909 (2004).

Acknowledgments. We acknowledge the use of data from SECCHI, IMPACT, PLASTIC, WAVES instruments on STEREO, LASCO on SOHO and WAVES on WIND. STEREO is the third mission in NASA's Solar Terrestrial Probes program, and $\mathrm{SOHO}$ is a mission of international cooperation between ESA and NASA. We thank anonymous referees for their constructive comments. Y.W. also thanks Noé Lugaz for some valuable discussion. This work is supported by grants from the 973 
key project 2011CB811403, NSFC 41131065, 40904046, 40874075, and 41121003, CAS the Key Research Program KZZD-EW-01-4, 100-talent program, KZCX2-YW-QN511 and startup fund, and MOEC 20113402110001 and the fundamental research funds for the central universities.

[Author Contributions] Y.W. designed the analysis of the collision and performed the theoretical derivations. C.S. found this event and made the data processing and calculations. S.W. gave constructive suggestions on the analysis of the collision. Y.L. gave some advice on the construction of the Jmap and interpretation of the elongation angle. B.M. carried out the literature investigation and provided valuable additions. A.V. calculated the masses of the CMEs and gave many valuable suggestions. R.L. and P.Y. participated in the discussion and gave many suggestive comments. J.L. and Z.Z. made a contribution to the data analysis.

[Author Information] The authors declare no competing financial interests. Correspondence and requests for materials should be addressed to Y.W. (ymwang@ustc.edu.cn). 